\title{
Optical Properties of Human Skin around Biological Active Points
}

\author{
R. Guzmán-Cabrera ${ }^{1 *}$, M. Vargas-Luna ${ }^{3}$, I. Delgadillo-Holtfort ${ }^{3}$, R. Huerta-Franco ${ }^{2}$, E. A. \\ Pérez-Alday ${ }^{1}$, T. Cordova-Fraga ${ }^{2}$ \\ ${ }^{1}$ Engineering Division, Campus Irapuato-Salamanca, University of Guanajuato, México. \\ 2 Department of Engineering Physics, University of Guanajuato, Campus León, México. \\ ${ }^{3}$ Department of Applied Science, University of Guanajuato, Campus León, México. \\ * Corresponding author. Email: guzmanc@ugto.mx \\ Manuscript submitted October 5, 2015; accepted February 12, 2016. \\ doi: 10.17706/ijcee.2016.8.1.57-65
}

\begin{abstract}
The physical parameter for the so-called Biologically Active Points most documented in the literature is the electrical impedance. These points seem to have higher electrical conductance than their surrounding points. The stimulation of these points is made not only mechanically and electrically but also optically (laser acupuncture) and thermically (moxibustion). We evaluated by optoacoustic technique and by photothermal radiometry, some skin points along the PC acupuncture meridian in the forearm region. We found a slight relative increment in absorption in some regions around the meridian region but without a definite association with an acupoint. Moreover, the same case can be applied to a non-meridian line. In radiometric signals we did not find differences with respect to control points in a two layer model. Based in these results we can not conclude that such points are optically or thermally special.
\end{abstract}

Key words: Acupuncture points, meridians, optical absorption, thermal properties.

\section{Introduction}

The acupuncture, being a very old methodology for diagnosis and therapy for health care, is still a controversial topic in research. Nevertheless, the National Institute of Health from the USA (1997) and the World Health Organization (2003) declared that there is enough clinical evidence to recognize the Acupuncture as an alternative treatment to prevent and cure diverse pathologies [1], [2].

The traditional form of stimulating Biological Active Points (BAP)'s or acupoints is by introducing needles into the skin in specific body points and performing mechanical stimulation mainly by needle rotation. Nowadays the BAP are stimulated also using others mechanisms as electrical stimulation yielding to the so called electro-acupuncture; thermal stimulation getting the method known as moxibustion; optical stimulation as in laser-acupuncture, and external mechanical stimulation known as acu-pressure [3], non mentioning magnetic stimulation used also in this alternative therapy [4].

In spite of the "acceptance" of acupuncture as an alternative therapy, the investigation related to its effectiveness and physical principle of action seems to be still not conclusive.

Many results have been published about clinical trials, testing the efficacy of the acupuncture as a therapy. The conclusions are controversial in the scientific community mainly among the occidental researchers [5]. The research on physical properties of these acupoints and their meridians is scarce and again controversial [6]. 
The physical properties of BAP more documented are the electrical properties, mainly electrical resistance or conductance. In fact, the usual BAP technique to localize acupoints uses the high conductance property by surface measurements. Even the electrical characterization of acupoints has generated controversy in scientific community because of the errors factors involved in the evaluation [7].

Very few work has been done in the optical and thermal properties of these points to support or at least to give some guide about the equivalence of the optical stimulation compared to the traditional mechanical stimulation. One of them deals with the reflectance properties of the points compared to sourrounded points [8] and two other works [9], [10] looked for infrared thermographic differences on the surface with controversial results. These works tried to get information about both the absorbance of the light beneath the surface and the thermal radiation from the surface. Another work about thermal phenomenon in acupuncture looked for possible electrostimulation through a temperature gradient in the needle [11].

In this work, we evaluated the relative magnitude of optical absorption of skin points by optoacustic technique using near infrared (NIR) low powered laser to penetrate into the near subcutaneous tissue and relative magnitude and phase of the photothermal radiometry (PTR) signal obtained from skin surface irradiated with a $514 \mathrm{~nm}$ diode laser. We took anatomical similar points as control references to have similar stratum-corneum, skin hydration, subcutaneous fat tissue etc. Individual BAP's and meridian line points were assessed and compared with their corresponding control points. Optoacustic and photothermal signal differences are discussed among all these points.

\section{Material and Methods}

\subsection{Experimental Set up}

Two BAP's were selected on the inner forearm skin PC4 (Ximen) and PC6 (Neiguan), some of the most used acupoints in scientific literature. The location of the acupoints was determined from traditional literature information without any acupuncturist guide and using an electrical locator. Using these two points and anatomical references we defined the "PC meridian" from wrist to 10 or $12 \mathrm{~cm}$ up to the forearm. The control line points were selected from a line parallel to the "meridian" around $1 \mathrm{~cm}$ toward the thumb avoiding cartilage and tendons tissue. Similar arm lengths and a roughly relation of one "cun" with $2 \mathrm{~cm}$ allow to have the PC acupoint with some mm of uncertainty but in any case each acupoint was localized with an electric device.

For optoacoustic assessments, Nd:YAG $1064 \mathrm{~nm}$ wavelength, 9 ns pulse duration, and $5 \mathrm{~Hz}$ repetition rate (Lotis TII LS-2130) was used to stimulate each point. An intensity of no more than $80 \mathrm{~mJ} / \mathrm{cm} 2$ was assured for safety reasons. The forearm was placed in water around $4 \mathrm{~cm}$ beneath the surface and a home-made PVDF sensor was used to detect the optoacustic signal (Fig. 1(a)). The signal was recorded in a 4 channel, 1 Gs/s oscilloscope (TDS5104B) and analyzed in MatLab.

For photothermal radiometric measurements a continum diode laser of less than $100 \mathrm{~mW}, 514 \mathrm{~nm}$ wavelength was used. Two off axis paraboloid mirrors collected, collimated and focused the black body IR radiation from the irradiated skin. The IR radiation was focused on a HgCdTe nitrogen cooled detector (Fig. 1(b)). The signal was recorded from a Lock-In amplifier by a PC in LabView.

The skin surface irradiated in both cases was placed beneath an aluminum plate with a circular hole (5 mm diameter) with minimum pressure to avoid skin surface curvature. The plate and hole also assured to detect signal coming from a small cone in the inner tissue beneath that point (Fig. 1(c)).

\subsection{Subjects}

For preliminary results about the feasibility of this experiment, we measured for optoacoustic evaluation two healthy volunteers 23 and 45 years old. For photothermal evaluation, 8 evaluations were performed on 4 healthy subjects. A general clinical check up was performed to each volunteer mainly to avoid skin and 
subcutaneous pathologies in the region of interest. Before participating in the experiment, each subject was abstained from smoking, alcohol consumption, strenuous exercise, and substances containing caffeine for 24 hours before testing and were asked to sleep enough the night before. Also, the volunteers were asked to arrive cleaned without cream, lotion, perfumed or any other cosmetic skin treatment. Before and after each optoacoustic or photothermal measurement, the skin was cleaned with alcohol. The subjects were recruited with a similar arm length to avoid uncertainty in the correlation of the localization of the measured points and the possible localization of the acupoints.

All subjects who participated in this study signed a consent form approved by the Medical Physics Group of the University of Guanajuato. The study was conducted according to the Declaration of Helsinki [12].

\subsection{Data Analysis}

The optoacoustic data were analyzed considering that the optoacoustic amplitude is proportional to the optical absorption coefficient for low and moderate absorption inside the skin [13]. The photothermal assessment consisted in the analysis of the measured phase lags of the different skin regions in terms of a two-layer sample model as in [14]. Only qualitative discussion on the results is presented due to the small sample considered.

\section{Results and Discussion}

\subsection{Optoacoustic Assessment}

Two subjects were measured along the PC meridian from the wrist (possible localization of the PC7 acupoint) toward the forearm every $5 \mathrm{~mm}$. The results are shown in Fig. 1(a)-(c) and Fig. 2 (a)-(d). The light peaks of the Fig. 1 correspond to the light regions of Fig. 2. In Fig. 2, the lines represent roughly the localization of the PC acupoints: skin point 8 for PC6, point 12 for PC5 and skin point 20 for possible PC6 position.

Fig. 1 shows a similitude in the absorption variation for both meridian and non-meridian, all along the line considered for the assessment. Roughly, the same peak of absorption observed at non-meridian is observed in meridian mainly in subject 1 . This is understandable because the non-meridian line was chosen near the meridian but with a similar anatomy and skin quality.

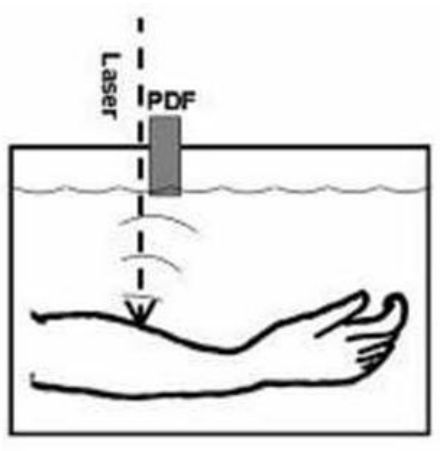

(a)

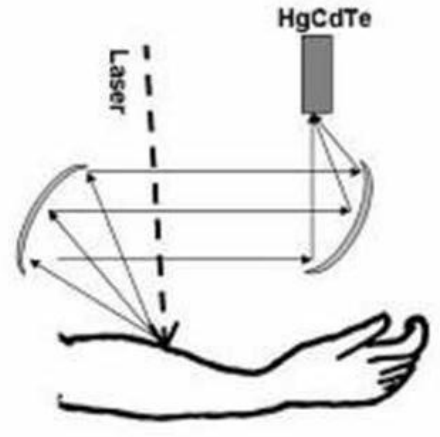

(b)

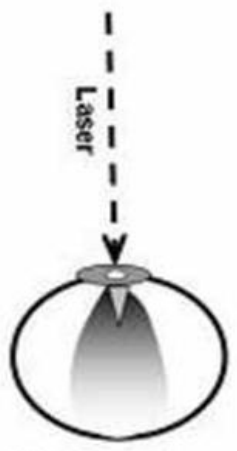

(c)

Fig. 1. Experimental setup for skin point evaluation a) Optoacoustic, and b) photothermal radiometry. The detected region is a cone near the skin surface c).

In Fig. 2, the localization of the signal for skin points 8, 12 and 20 can be related to the light peaks more easily. The skin position 8, supposedly belonging to PC6 at PC meridian does not show a significant enlighten peak except in the non-meridian graph for subject 1 . The meridian graphs do not show a special absorption characteristic at this position. It is worth mentioning that PC6 is one of the acupoints most mentioned in clinical trials in scientific works. The skin position 12 , supposedly belonging to PC5, show a 
peak in both subjects in meridian lines and a very smooth peak in non-meridian of subject 1.

Finally the skin point 20 (not evaluated in non meridian of subject 1) would correspond to PC4, maybe with more uncertainty because of the small differences in arm length for both subjects, could show a special bright region in the non-meridian evaluation of subject 2.

A scanning at different depths (not only looking for signal maximum but trying point averages, depth interval averages or global averages) seems not to improve these findings about (i) the similarity in the variability of optical absorption for PC meridian and anatomically similar non-meridian line and (ii) the very poor correlation of maximum of absorption and roughly position of PC acupoints. There is the possibility of having missed the real acupoints in meridian evaluation because of the $5 \mathrm{~mm}$ step scan like a similar dielectric scan seems to indicate in a previous work [15]. This would imply that acupoints are points (actually skin regions or spots) with very small dimensions. Other possibility is that optical obsorption could not be the physical parameter that would characterize the acupoints, but rather the optical stimulation should be associated in some way to the known electrical differences of these points with respect to their surroundings skin points.

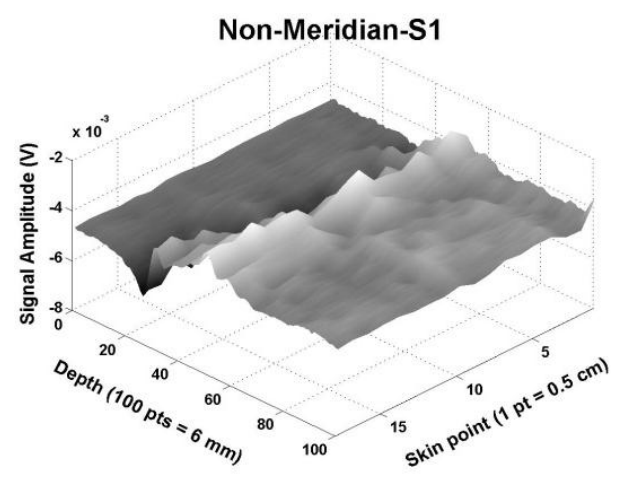

(a)

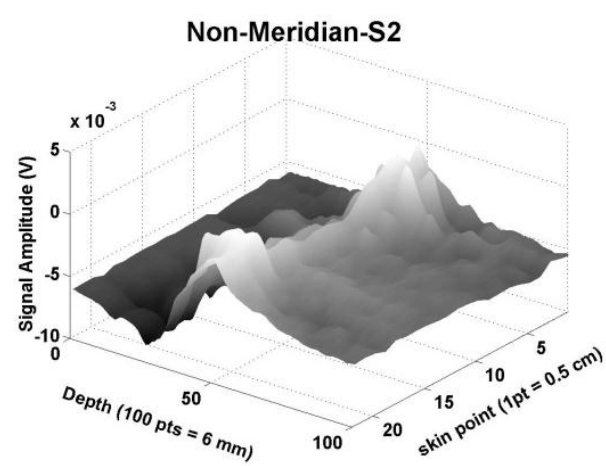

(c)

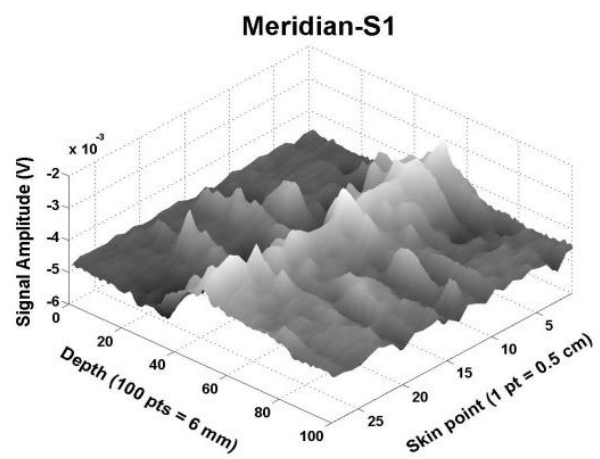

(b)

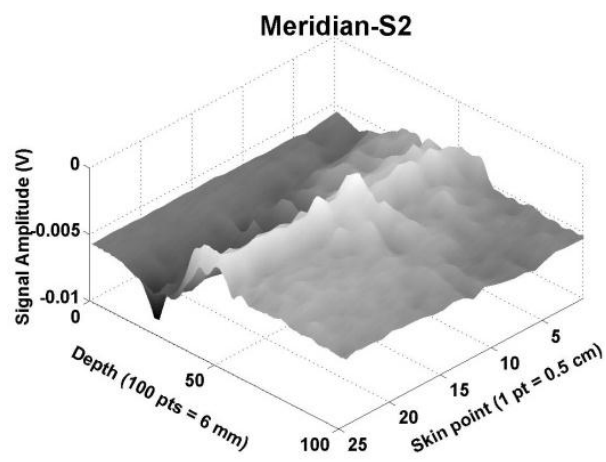

(d)

Fig. 2. Optoacoustic signal for skin points separated $0.5 \mathrm{~cm}$ from each other starting from the wrist. The signal amplitude corresponds to around $5 \mathrm{~mm}$ depth inside each point. The signal is for (a) non-meridian line in subject S1, (b) PC meridian line in subject S1, (c) non-meridian line in subject S2 and (d) PC meridian line in subject $\mathrm{S} 2$.

\subsection{Photothermal Radiometry Assessment}

Radiometric evaluation was performed in four subjects performing eight evaluations of three points, two of them supposedly corresponding to PC6 and PC4 acupoints and one PC meridian point not associated with an acupoint in the middle of the two acupoints mentioned, so that we will call it in the dollowing M46. Each point was accompanied with its corresponding control point localized nearby in an anatomically similar region with similar skin quality (see Fig. 3). 


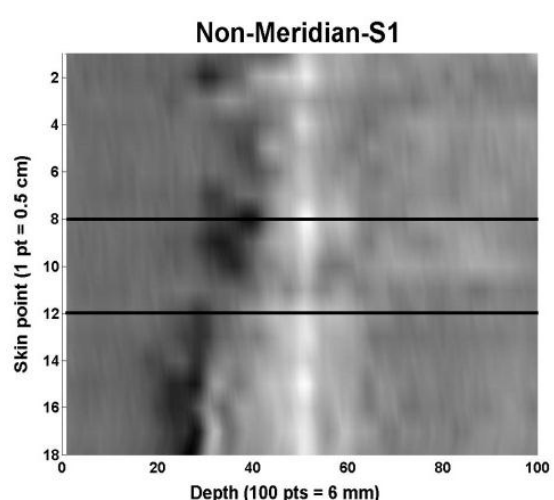

(a)

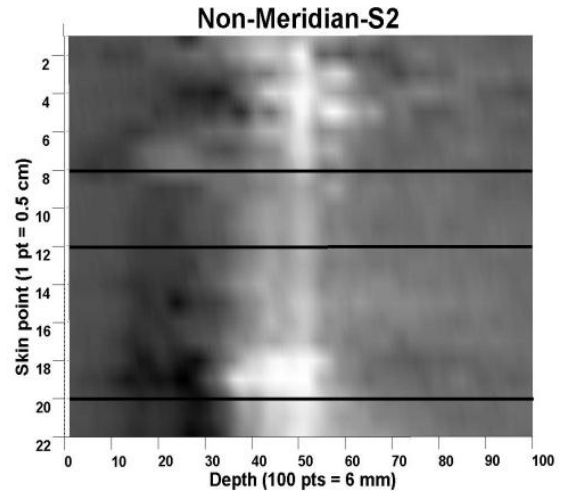

(c)

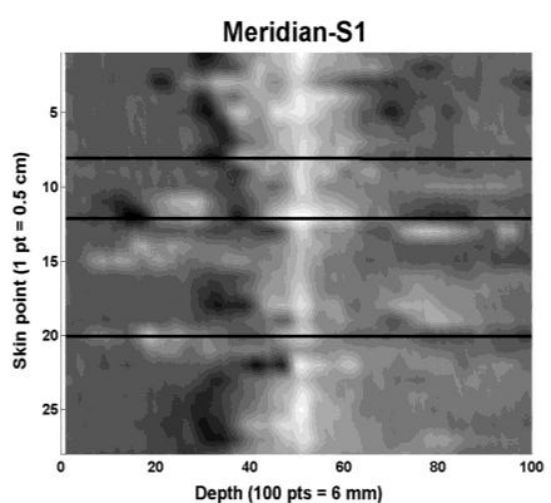

(b)

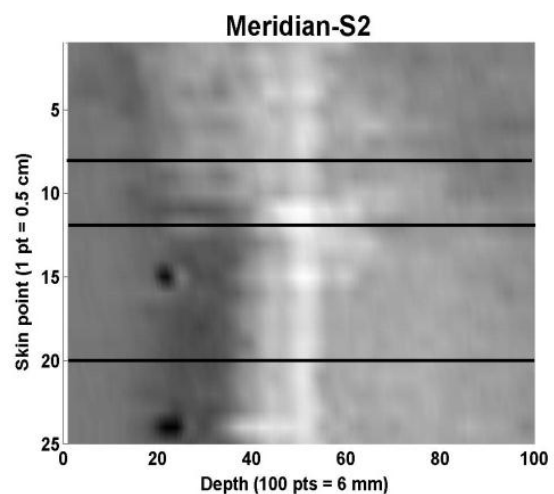

(d)

Fig. 3. Optoacoustic signal in normal view. The lines correspond roughly to the possible position of the PC acupoints. Line in skin point position 8 would correspond to PC6 acupoint, that in position 12 to the PC5 and the one in position 20 for PC6. The signal is for (a) non-meridian line in subject 1, (b) PC meridian line in subject 1, (c) non-meridian line in subject 2 and (d) PC meridian line in subject 2.

Fig. 4 shows the raw frequency dependent PTR-signal obtained for one of the subjects for all mentioned points. The measured modulated IR amplitudes and phase lags are usually affected by the frequency characteristics of the measuring system. This can be eliminated when inverse normalization to an appropriate reference material, i.e., reference-to-sample normalization, is taken. For this reason, the signal for glassy carbon, a homogeneous opaque reference material, is also measured and the corresponding signal is also shown in Fig. 4.

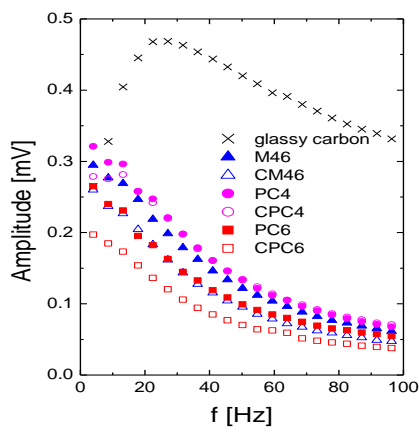

(a)

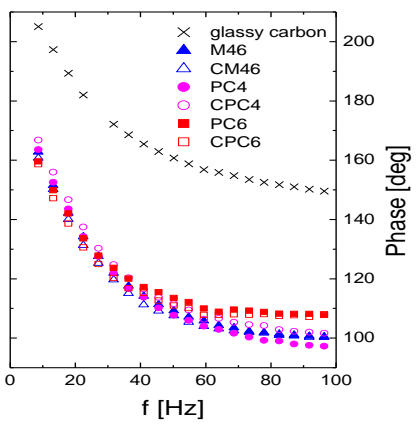

(b)

Fig. 4. Photothermal radiometry (a) amplitudes and (b) phases as a function of the modulation frequency $\mathrm{f}$ obtained for one subject at the biological active points PC4 and PC6, and meridian point in between (M46) not corresponding to PC5. The signals of the corresponding control points CPC4, CPC6 and CM46, as well as the signal obtained for glassy carbon are also shown. 
The inverse normalization is also a suitable tool to perform thermal properties depth profiling of layered samples (s. e.g. [16] or [17]). Particularly, within consideration of a two-layer sample model the use of the normalized phase lags as a function of the square root of the modulation frequency allows the univocal determination of combined thermophysical parameters through the determination of the normalized signal phase extremum (value and frequency position) that is obtained for this kind of samples.

These combined thermophysical parameters are (i) the ratio of the thermal effusivities surface layer-to-substrate, and (ii) the thermal diffusion time of the surface layer, which is defined as the square of the thickness of the surface layer divided by its thermal diffusivity value [14]. Therefore, comparison of the normalized phase lags extrema (values and frequency values) is an easy way to assess the existence of statistically significant differences between such combined thermophysical parameters of two-layered samples.

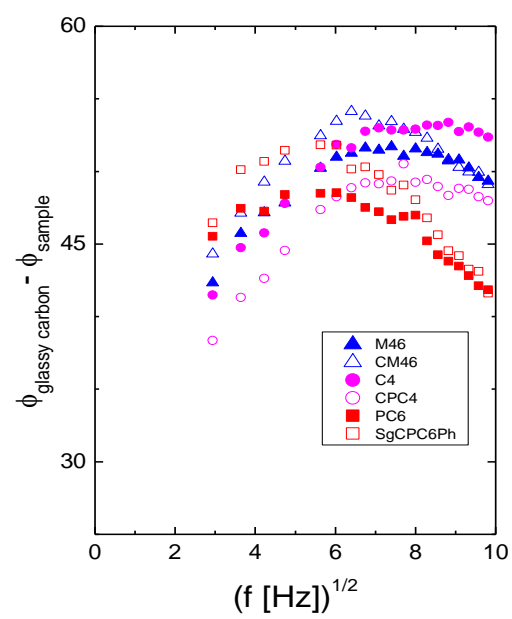

Fig. 5. Inverse normalized IR phases obtained for one subject at the biological active points PC4 and PC6, and meridian point in between (M46) not corresponding to PC5, as well as for the corresponding control points CPC4, CPC6 and CM46. The inverse normalization has been perfomed using glassy carbon as reference material.

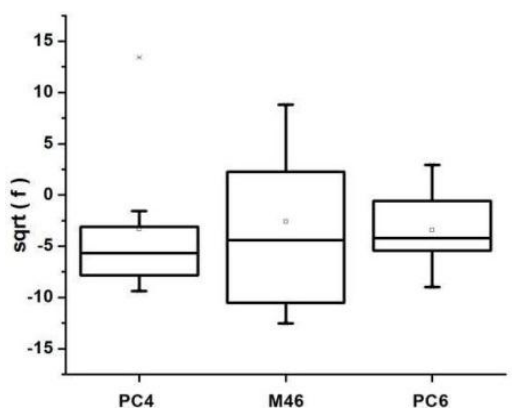

(a)

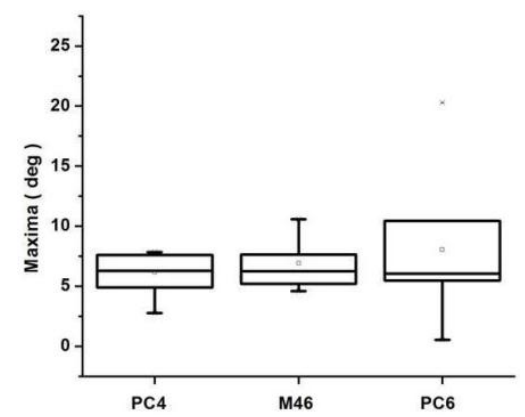

(b)

Fig. 6. Square root frequencies at which the maxima of the inverse normalized phase signal of PC4, PC6 and M46 are positioned (a) and (b) values of these maxima.

In Fig. 5, we show the normalized photothermal phases of PC6, PC4 and M46 and the control points as a function of square root of the modulation frequency. The normalization has been performed taking glassy carbon as normalizer. It has to be noted that the signals do present one defined maximum and that all maxima are distinct, so that different values for the combined thermophysical properties are expected. However, statistical analysis run for the eight measurements performed for each skin region yield to the result shown in Fig. 6. In this figure, we see that for the skin regions considered there are not any significant 
differences either between the square root frequencies at which the extrema are located at or between these extrema values. This points out that at least within the two-layer model considered the thermophysical parameters of BAps and non-BAPs for similar skin quality are not significantly different.

\section{Conclusions}

The optoacoustic methodology used to measure optical absorption of skin and subcutaneous tissue assured that the similarities or differences founded are not only due to skin surface differences but also includes possible nearby subcutaneous differences. The variability of the absorption is similar in meridian than in a parallel non-meridian line along the forearm. There exist localized regions of high absorption but hardly associated with acupoints unless we had missed the acupoints due to the $5 \mathrm{~mm}$ step that could be huge in a hypothetical very punctual region that could define acupoints. The thermal properties do not seem likely to be useful to discriminate acupoints from their surrounding points.

Statistical analysis with more subjects could be performed together with a very fine 2D-net of evaluated points near the meridian to discard the missing of possible very localized real points used in acupuncture.

\section{References}

[1] National Institutes of Health. (1997). Consensus development statement. Acupuncture, 15(5), 1-34.

[2] WHO. (2003). Review and analysis of reports on controlled clinical trials. Acupuncture.

[3] Stux, G., Berman, B., \& Pomeranz, B. (2003). Basics of Acupuncture (5th ed.). Springer.

[4] Yang, S., Xu, G., Geng, Y., Ho, S. L., Wang, L., \& Zhang, X. (2008). Biomagnetism - Transdisciplinary Research and Exploration.

[5] Ernst, E. (2006). Acupuncture - a critical analysis. J. Intern. Med, 259(2), 125-137.

[6] Ahn, A., Colbert, A., Anderson, B., Martinsen, O., Hammerschlag, R., Cina, S., Wayne, P., \& Langevin, H. (2008). Electrical properties of acupuncture points and meridians: A systematic review. Bioelectromagnetics, 29(4), 245-256.

[7] Ahn, A., \& Martinsen, O. (2007). Electrical characterization of acupuncture points: Technical issues and challenges. J. Altern. Complement. Med., 13(8), 817-824.

[8] Yang, H., Xie, S., Wang, Y., \& Lu, Z. (2006). Nanophotonics and Metamaterials.

[9] Litscher, G. (2005). Infrared thermography fails to visualize stimulation-induced meridian-like structures. Biomed. Eng., 4(38).

[10] Narongpunt, V., Cornillot, P., Attali, J., Molinier, F., Alimi, D., Datcu, S., Ibos, L., Candau, Y., Fontas, B., Raji, A., Clairac, B., Bloch Danan, S., \& Marignan, M. (2005). Infrared thermographic visualization of the traditional Chinese acupuncture meridian points. Medical Acupuncture, 16(2), 32-37.

[11] Cohen, M., Kwok, G., \& Cosic, I. (1997). Acupuncture needles and the seebeck effect: Do temperature gradients produce electrostimulation? Acupunct. Electrother. Res., 22(1), 9-15.

[12] WMA News. (2008). Revising the declaration of Helsinki. World Medical Journal, 54, 120.

[13] Karabutov, A., Savateeva, E., Podymova, N., \& Oraevsky, A. (2000). Backward mode detection of laser-induced wide-band ultrasonic transients with optoacoustic transducer. J. Appl. Phys., 87, 2003.

[14] Nzodoum-Fotsing, J. L., Gibkes, J., Pelzl, J., \& Bein, B. K. (2005). Extremum method: Inverse solution of the two-layer thermal wave problem. J. Appl. Phys., 98(6), 063522.

[15] Vargas-Luna, M., Perez-Alday, E., Huerta-Franco, R., \& Delgadillo-Holtfort, I. (2009). Optical properties of human skin around biological active points. Int. J. Bioelectromagnetism.

[16] Delgadillo-Holtfort, I., Pelzl, J., \& Bein, B. K. (2002). Control of drying by means of IR detection of thermal waves. Quantitative Infrared Thermography, 5, 230-235.

[17] Gibkes, J., et al. (2001). Photothermal characterisation of local and depth-dependent thermal properties of NiTi shape-memory alloys. Proceedings of Mater. Sci. Forum (pp. 345-348, 394-395). 


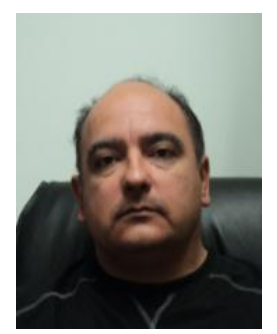

R. Guzmán-Cabrera is a titular researcher at Universidad de Guanajuato for 17 years, he received a PhD degree in pattern recognition and artificial intelligence from Polytechnic University of Valencia, Spain. He is a member of the National System of Researchers of the National Council of Science and Technology of Mexico. And also is a contributor in research projects in the area of electrical engineering, pattern recognition and artificial intelligence.

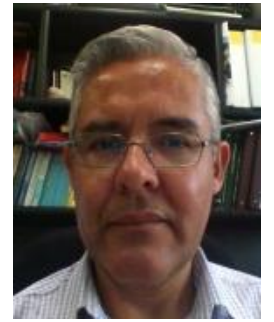

F.-M. Vargas-Luna is a full time professor at the University of Guanajuato, member of the National Research System (Mexico) since 1990 and of the Mexican Academy of Science since 2005. His main research topics include biomedical applications of electrical bio-impedance and signal processing (EGG, ECG, PPG). He has 64 international publications, 1 book chapter, 2 edited books, 9 refereed proceedings and 42 non-refereed proceedings. He is a teacher of physics and mathematical courses at undergraduate and graduate level. He had been the academic secretary at the Physical Institute University of Guanajuato from 1997 to 2000, the director of the Applied Physics Department from 1997 to 2006, and the director of the Physical Engineering Department from 2008 to 2012.

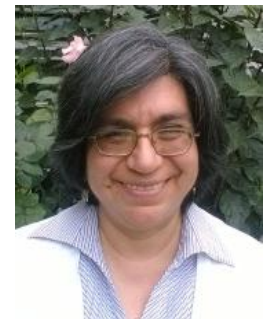

I. Delgadillo Cano has been with University of Guanajuato, Sciences and Engineering Division, Campus León, Department of Physical Engineering since 2006. He is now an associate professor.

He received the bachelor degree in physics and mathematics (specialty in physics), from Escuela Superior de Física y Matemáticas, National Polytechnic Institute (IPN), Mexico, in 1986. Then received his master in sciences degree (specialty in physics), from Center for Research and Advanced Studies of the National Polytechnic Institute (CINVESTAV-IPN) in 1988; and the PhD degree (specialty in physics), from CINVESTAV-IPN in cooperation with the University of Duisburg "Gerhard-Mercator", in 1994. He did his postdoctoral research at Switzerland (ETH-Zürich) and Germany (Ruhr-Universitaet Bochum).

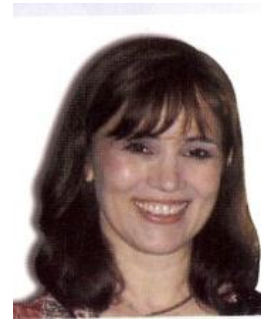

R. Huerta-Franco has a PhD degree in medical sciences, she is a titular B professor at University of Guanajuato since 1991. She is a member of the National System of Researchers of the National Council of Science and Technology of Mexico since 1992. She did her post-doctorate at University of Alabama at Birmingham from 1998 to 1999, in the area of reproductive endocrinology. She did research in the Department of Psychology at the University of Rotgers, New Jersey, from 2006 to 2007. She is a editorial board member of World Journal of Gastroenterology Pathophysiology, Beijing, from 2010 to 2015. She also has the recognition by the Ministry of Health of the State of Guanajuato, as a distinguished research professor in 2008. She was the president of the Academy for Research in Reproductive Biology, from 1999 to 2000. She received the Alfonso Rivera Award in 1996, as a young leading researcher and issued by the Mexican Society of Nutrition and Endocrinology.

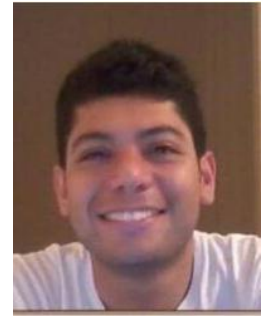

E. A. Perez-Alday has a master in sciences degree of theoretical and applied physics, from Institute of Physics, University of Guanajuato, Mexico, in collaboration with the Universitat Politecnica de Catalunya, Barcelona, Spain. His main research interests are in applying physics and math to problems arising in medicine and biology, with the aim to improve monitoring, imaging and diagnosis of cardiovascular diseases. He has research experience in cardiac modelling and imaging to improve non-invasive methods for 
monitoring and diagnose the electrical activity of the heart, as well as in skin electric and photoacoustic measurements, electro impedance tomography (EIT) measurements to calculate the lungs ventilatory patterns.

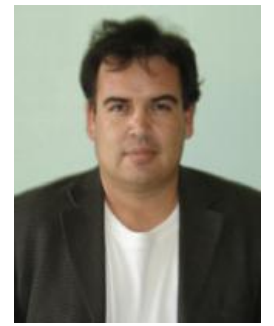

Teodoro Cordova-Fraga received the degree in physics-mathematics from Universidad Autonoma de San Luis Potosi, SLP, Mexico in 1997 and the MSc. and PhD degrees from Universidad de Guanajuato, GTO, Mexico in 2001 and 2003, respectively. He spent a year during his PhD research in the Biomagnetism Laboratory of Universidade de São Paulo, Campus Ribeirão Preto in Brazil. He was a research fellow in the Department of General Surgery at Vanderbilt University, Nashville, TN, USA, from August 2004 to July 2005. Currently, he is performing studies of Gl's biomagnetism in animal and humans. His current interests include magnetobiology, biomedical instrumentations, bioelectromagnetic detection of the human body, ultrasound and the images processing. He is a member of the Mexican Society of Bioengineering and the Medical Physics Divisón of the Mexican Society of Physics. 